\title{
EL COORDINADOR DE PARENTALIDAD: UN ANÁLISIS DE LAS RESOLUCIONES JUDICIALES EN ESPAÑA
}

\author{
PARENTING COORDINATOR: AN ANALYSIS \\ OF SPANISH JUDICIAL REASONING
}

\author{
FRANCISCA FARIÑA ${ }^{1}$, VANESA PARADA ${ }^{2}$, MERCEDES NOVO $^{3} \mathrm{Y}^{\mathrm{N}}$ \\ DOLORES SEIJO ${ }^{3}$
}

Cómo referenciar este artículo/How to reference this article:

Fariña, F., Parada, V., Novo, M. y Seijo, M. (2017). El Coordinador de Parentalidad: Un análisis de las resoluciones judiciales en España [Parenting Coordinator: An Analysis of Spanish Judicial Reasoning]. Acción Psicológica, 14(2), 157-170. http://dx.doi.org/10.5944/ap.14.1.21346

\section{Resumen}

El coordinador de parentalidad (CP) interviene en situaciones de judicialización de la relación entre los progenitores y de alto conflicto, abordando desde el interés superior de los menores, las cuestiones relativas a la coparentalidad. Este nuevo rol ha sido objeto de directrices específicas de las asociaciones profesionales internacionales, tales como Association of Family and Conciliation Court (AFCC, 2006) y la American Psychological Association (APA, 2012), en las que se establecen orientaciones para la práctica de este profesional. En España, su implantación es muy reciente, siendo iniciada en Cataluña y seguida por otras comunidades autónomas. Desde este contexto, nos planteamos un estudio para conocer cómo se ha concretado esta nueva figura en las resoluciones judiciales, atendiendo por una parte a la motivación legal $\mathrm{y}$, por otra, a la motivación de la práctica que los jueces atribuyen a esta figura. Se llevó a cabo un análisis de contenido de las resoluciones judiciales que incluían la figura de Coordinación de Parentalidad en la base de datos CENDOJ. Los resultados obtenidos permiten conocer los detalles legales que los decisores judiciales incluyen en sus resoluciones, así como los criterios que emplean para motivar esta figura y su correspondencia con las directrices internacionales. Se concluye que las sentencias se dotan de motivación legal, y que los criterios se corresponden, en lo esencial, con las directrices editadas por las asociaciones internacionales, aunque sería preciso mejorar el conocimiento de los

Corresponding author: Francisca Fariña. Facultade de Ciencias da Educación e do Deporte.

Campus a Xunqueira, Pontevedra, España. Email: francisca@uvigo.es

ORCID: Francisca Fariña (https://orcid.org/0000-0002-7652-0948) y Mercedes Novo (https://orcid.org/0000-0002-0942$\underline{1501)}$

${ }^{1}$ Departamento AIPSE, Universidade de Vigo, España.

${ }^{2}$ Unidade de Psicoloxía Forense, Universidade de Santiago de Compostela, España.

${ }^{3}$ Departamento de Psicoloxía e Socioloxía, Universidade de Santiago de Compostela, España.

Recibido: 10 julio de 2017.

Aceptado: 18 de septiembre de 2017. 
operadores jurídicos acerca de las posibilidades que ofrece la $\mathrm{CP}$, para ayudar a las familias con separaciones conflictivas de los progenitores a ejercer una coparentalidad positiva.

Palabras clave: Coordinador de parentalidad; Divorcio; Ruptura de pareja; Resoluciones judiciales.

\begin{abstract}
A parenting coordinator (PC) is a professional who intervenes in judicial contexts involving high conflict parental litigation in order to safeguard the child's best interest, as well as dealing with issues concerning coparenting. This new role has been the object of specific directives from international professional associations such as the Association of Family and Conciliation Courts (AFCC, 2006), and the American Psychological Association (APA, 2012), who have established guidelines for professional practice. The figure of the PC has been introduced only recently in Spain, with Catalonia being the first autonomous communities to do so. Bearing these circumstances in mind, this study aims to assess the impact of this new figure on judicial judgements by examining both the legal reasoning, and the judges' rationale guiding the professional practice of this figure. Judicial judgements from the CENDOJ database involving the figure of the Parenting coordinator were analysed. The results obtained shed light on the legal reasoning cited by judicial decision-makers in their judgements and the criteria employed to justify this new figure and its correspondence with international guidelines. Judgements were found to be grounded on legal reasoning and the criteria was essentially in accordance with guidelines proposed by international associations. Nevertheless, legal decisionmakers must further their understanding concerning the role of the CP in order to assist families with high conflict parents to ensure positive coparenting.
\end{abstract}

Keywords: Parenting coordinator; Divorce; Couple breakdown; Child's best interest; Judicial judgements.

\section{Introducción}

La coordinación de parentalidad (CP), denominada de diferentes maneras, tales como special master, wiseperson, family court advisor, mediator-arbitrator, o parenting plan coordinator (Sullivan, 2013), tiene sus orígenes a mediados de los años ochenta del siglo pasado en Estados Unidos (Kelly, 2014). A inicios de los 90, la CP surge como un nuevo procedimiento ADR (Carter, 2011) para resolver los casos altamente conflictivos de ruptura de pareja con hijos menores de edad. A este respecto, se mencionan como pioneros de la CP los estados de California y Colorado (Kelly, 2014; Sullivan, 2013), siendo Oklahoma, en 2001, el primer estado en desarrollar una norma legal para darle soporte (Kelly, 2014), siguiéndole rápidamente otros en conferirle una regulación estatutaria ad hoc (Brown, Behrman, y Zimmerman, 2017; Montiel, 2015). Si bien no todos los estados cuentan con dicha regulación (Ordway, 2015), actualmente esta práctica se encuentra asentada en todo el territorio norteamericano, tanto en Canadá como en Estados Unidos, siendo muchos los estados en los que se ha implantado activamente (Behrman, 2016; Neff y Cooper, 2004; Parks, Tindall y Yingling, 2011).

La coyuntura en la que surge la $\mathrm{CP}$ se caracteriza por un elevado número de divorcios, una creciente demanda de la custodia compartida, y el uso principal de la vía judicial, por parte de algunos progenitores, para solventar las cuestiones relativas al plan de parentalidad, a lo que había que añadir que las sentencias judiciales no resolvían el problema, produciéndose una judicialización de la relación parental, con el subsecuente daño a los hijos (Kelly, 2014). Se estima que entre el $8 \%$ y el $12 \%$ de los progenitores con ruptura de pareja continúan con un nivel alto de conflicto (Coates, Deutsch, Starnes, Sullivan, y Sydlik, 2004). Las situaciones de "alto conflicto" hacen referencia a las disputas intensas que se prolongan en el tiempo, que requieren considerables recursos judiciales y comunitarios, y que se caracterizan por falta de confianza entre los progenitores, elevados niveles de enfado entre ellos, incapacidad para comunicar sus sentimientos y necesidades de manera apropiada y uso frecuente del sistema judicial (American Bar Association, 2005; Coates et al., 2004; D’Abate, 2016; Kelly, 2003; Shaw, 2017). A pesar de que el porcentaje de casos pue- 
da parecer escaso representan un desafío para el sistema judicial y para los profesionales que trabajan en el campo de la salud mental y legal; consumiendo, además, una enorme cantidad de tiempo de los tribunales y servicios legales gratuitos, y de la mayor parte de los escasos servicios psicosociales (D’Abate, 2016; Saini y Birnbaum, 2007); se estima que ocupan el $90 \%$ del tiempo de los jueces de familia y de los profesionales implicados (Neff y Cooper, 2004). Esto es, utilizan una parte desproporcionada tanto del tiempo como de los recursos de los juzgados (Mitcham-Smith y Henry, 2007). Esto podría ser suficiente para motivar la búsqueda de procedimientos que ayuden a pacificar estas familias; sin embargo, resta el daño más importante, el que se causa a los hijos (Corrás et al., 2017; Martinón et al., 2017; Seijo, Fariña, Corrás, Novo y Arce, 2016).

La CP es definida por la Association of Families and Conciliation Court (AFCC, 2006) como un proceso alternativo de resolución de conflictos centrado en los niños, en el que un profesional de la salud mental o del ámbito legal, con formación y experiencia en mediación, asiste a los padres que presentan alta conflictividad y les enseña a implementar el plan de parentalidad sin dañar a sus hijos. En la misma línea, la American Psychological Association (APA, 2012) establece que la CP es un proceso de resolución de conflictos de carácter no adversarial, que puede ser ordenado por el juzgado o acordado por los propios padres, quienes están separados o divorciados y sumidos en un continuo conflicto o litigio, que afecta negativamente a la relación con sus hijos. La CP se considera como un modelo de intervención desde el que abordar las cuestiones de coparentalidad que surgen después de la separación o divorcio (Boyan y Termini, 2005; Fidler, 2012; Henry, Mitcham y Henry, 2013; Henry, Fieldstone y Bohac, 2009), cuyo principio rector de actuación es el mejor interés de los hijos y que cumple con los principios que definen el paradigma de la Justicia Terapéutica (TJ) (Fariña, Novo, Arce y Vázquez, 2017).

Para orientar la práctica de la $\mathrm{CP}$, las directrices publicadas por la AFCC (2006) y la APA (2012) precisan aspectos como la designación del $\mathrm{CP}$, sus funciones $\mathrm{y}$ objetivos, el proceso de intervención, así como la formación que han de poseer estos profesionales. Así, en relación a la designación se especifica que el CP puede ejer- cer su función por acuerdo entre los progenitores y/o por resolución judicial, debiendo concretarse y definirse con claridad su ámbito de autoridad y sus responsabilidades. El profesional precisa que bien los propios progenitores, a través del consentimiento informado o bien el tribunal, por orden judicial (Coates et al., 2004; Deutsch y Lally, 2014) le otorguen autoridad. Esta es necesaria para obtener toda la información que requiera el caso para gestionar el conflicto, tomar decisiones relacionadas con la satisfacción de las necesidades de desarrollo y psicológicas de los hijos, a la vez que desarrollar labores de carácter educativo con ambos progenitores, todo ello con el propósito de reducir en los hijos el daño que les produce la exposición al conflicto y eliminar o cuando menos minimizar el litigio entre los progenitores en cuestiones relacionadas con la parentalidad (AFCC, 2006; APA, 2012). Esta autoridad no puede ser limitada, puesto que de serlo reduce la eficacia de la intervención (Mandarino, Kline y Fieldstone, 2016), alcanzando así la posibilidad de arbitrar cuando los progenitores no se ponen de acuerdo. Sin la autoridad para arbitrar, es mucho más probable que la CP fracase, y los progenitores reinicien el litigio (Demby, 2016). Sin embargo, esta autoridad ha de estar delimitada. En este sentido, cuando se interviene dentro de un proceso judicial, la autoridad del CP se encuentra circunscrita a cuestiones cotidianas que pueden demandar una decisión rápida, dejando al margen aquellas de gran alcance que únicamente podrá tomar el juez (Carter, 2011; Emery, Rowen y Dinescu, 2014). Además, el tiempo de intervención del CP debería prefijarse en el mandato judicial. Al depender de la complejidad del caso y del modelo de CP que vaya a aplicarse resulta algo difícil de establecer, aunque muchos tribunales la limitan a uno o dos años (Carter, 2011).

En relación a las funciones atribuidas al $\mathrm{CP}$, la AFCC (2006) señala: (a) función de educación (enseña y puede entrenar a los progenitores respecto al desarrollo del menor, el impacto de su comportamiento en los menores, en habilidades parentales y unificación de pautas educativas, destrezas de comunicación, resolución de conflictos, etc.); (b) función de evaluación (tiene en cuenta cualquier información relevante del caso como las resoluciones judiciales, o de haberse practicado la evaluación pericial de custodia; además, revisa la información obtenida de las entrevistas con los progenitores, los menores 
o cualquier otra fuente de información colateral, como la familia extensa o el colegio, analiza los impedimentos y problemas expuestos por las partes); (c) función de gestión del caso (trabaja y se coordina con otros profesionales del ámbito de la salud, educación, servicios sociales o jurídicos que estén implicados con la familia así como con la familia extensa, nuevas parejas y otras personas significativas); (d) función de gestión de conflictos (ayuda a los progenitores a minimizar el conflicto y a resolver los desacuerdos relativos a sus hijos de manera que éstos no le causen daño); y (e) función de toma de decisiones (cuando los progenitores no son capaces de ponerse de acuerdo sobre cuestiones del día a día, el CP arbitra la solución). Se trata por tanto de un rol híbrido (Deutsch, 2014) que siempre se lleva a cabo en situaciones complejas caracterizadas por un alto nivel de conflicto (Carter, 2011) y de carácter persistente relacionado con la coparentalidad, o con la existencia de un historial de violencia familiar (D'Abate, 2005, 2016), con exclusión de aquellos supuestos de violencia familiar activa o enfermedad mental severa (Fariña et al., 2017; Sullivan, 2013). Este contexto requiere que el $\mathrm{CP}$ disponga, de manera acreditada, de las competencias profesionales necesarias para trabajar en el ámbito de la intervención con familias en conflicto y crisis, así como experiencia en esta área (Ke1ly, 2014; Kirkland y Sullivan, 2008; Lally y Deutsch, 2014; Sullivan, 2004). Tanto la AFCC (2006) como la APA (2012) también han delimitado en sus directrices, las áreas de formación del CP, las cuales incluyen conocimientos sobre: (a) el impacto de la ruptura de la pareja tanto en los progenitores como en los hijos, así como de los factores de riesgo y protección que pueden influir en el ajuste de todos los miembros de la familia tras la ruptura; (b) las dinámicas familiares en procesos de ruptura (v.gr., negativa de los hijos a acompañar a alguno de los progenitores, alto nivel de conflicto entre los progenitores); (c) las situaciones y problemáticas específicas como violencia de género o maltrato infantil; (d) la terminología legal y las leyes sobre materia de derecho de familia, de violencia doméstica y de género y sobre protección de menores; (e) formación en planes de coordinación de parentalidad y en procedimientos judiciales específicos de coordinación de parentalidad; y (f) técnicas y estrategias de mediación familiar.
La CP como ya se ha señalado, es una figura reconocida en Estados Unidos y Canadá (D’Abate, 2016). Fuera de estos países, la CP está menos instaurada, si bien existen iniciativas en Europa y Australia (Kelly, 2014). En España, aunque no contamos con regulación legal de la CP (Rodríguez-Domínguez y Carbonell, 2014), existe alguna experiencia, puesto que los tribunales que llevan asuntos de familia han de realizar el seguimiento de las medidas establecidas en las sentencias y resoluciones judiciales, en especial cuando existe una alta conflictividad en las relaciones parentales, y de no cumplirse, se han de adoptar medidas ad hoc (Ortuño, 2013). La primera comunidad autónoma que implantó judicialmente esta práctica fue Cataluña, siendo su principal promotor el magistrado Pascual Ortuño (Avedillo, Carrasco, Guitart y Sacasas, 2015), iniciativa que ha sido seguida por otras comunidades autónomas (p.ej., Aragón, Galicia, Madrid, Navarra, Valencia), dando lugar a un aumento del número de sentencias que dictan la designación de un $\mathrm{CP}$ (Capdevila, 2016). La situación actual en España es similar a la existente en Estado Unidos en los años 90, a lo que hay que añadir las directrices marcadas desde Europa instando a la igualdad y la promoción del ejercicio equitativo de la parentalidad y que ambos progenitores han de asumir, de modo compartido, la responsabilidad sobre sus hijos (Consejo de Europa, 2015).

Desde este contexto, y teniendo en cuenta la trayectoria de la CP en España, se plantea este trabajo con dos objetivos. Por una parte, considerando criterios empíricos, analizar las bases legales en las que se sustenta el mandato judicial de CP. Por otra, revisar el impacto de las directrices internacionales de la AFCC (2006) y la APA (2012) en las resoluciones judiciales, en cuanto al objeto de la intervención, la formación que se requiere, las funciones que ha de realizar, y a la designación e implementación de la CP, con el fin de analizar el ajuste de los mandatos judiciales en España a estas directrices. 


\section{Método}

\section{Protocolos}

Los protocolos de resoluciones judiciales se han obtenido de la base de datos del Centro de Documentación Judicial (CENDOJ) del Consejo General del Poder Judicial, la cual proporciona acceso a todas las resoluciones procedentes de tribunales colegiados del territorio nacional. Los términos empleados en la búsqueda fueron Coordinación de Parentalidad, Coordinador Parental, y Coordinación Parental. Una primera búsqueda general proporcionó 672 resoluciones judiciales. Posteriormente, se procedió a eliminar 564 que, o bien no se referían a la figura del coordinador de parentalidad (un total de 265), o bien se trataba de protocolos que aparecían duplicados en las diferentes búsquedas (un total de 299), resultando 108 resoluciones. Se aplicó un segundo filtro que descartó 33 resoluciones en base a las siguientes causas: (a) únicamente se menciona la intervención del coordinador como una opción entre otras a la que los cónyuges pue- den acudir (nueve protocolos), (b) no se menciona la intervención del coordinador de parentalidad en el fallo de la sentencia ni se aportan datos sobre su intervención en el fundamento o en los antecedentes (15 protocolos), (c) alguno de los litigantes solicita la intervención del coordinador de parentalidad, pero no se aportan más datos acerca de la misma (cuatro protocolos), (d) se menciona que ya ha intervenido un coordinador de parentalidad con la familia pero no se aporta nada más (tres protocolos), y (e) se eliminan dos autos aclaratorios.

Finalmente, la muestra quedó conformada por 75 resoluciones judiciales que cronológicamente estaban comprendidas entre julio de 2012 y noviembre de 2017. En la Tabla 1 se muestran los análisis descriptivos de las resoluciones, pudiendo constatarse que principalmente son sentencias, dictadas por la Audiencia Provincial de Barcelona, que provienen de Juzgados de Primera Instancia, mayoritariamente en asuntos de divorcio o de modificación de medidas.

Tabla 1

Descriptivos de las resoluciones judiciales

\begin{tabular}{|c|c|c|}
\hline Variables & Categoría & $f(\%)$ \\
\hline Tipo de resolución judicial & $\begin{array}{l}\text { Sentencias } \\
\text { Autos }\end{array}$ & $\begin{array}{c}71(94.6) \\
4(5.3)\end{array}$ \\
\hline Procedencia territorial & $\begin{array}{l}\text { Barcelona } \\
\text { Palma de Mallorca } \\
\text { Málaga } \\
\text { Lleida } \\
\text { Girona }\end{array}$ & $\begin{array}{l}71(94.6) \\
1(1.3) \\
1(1.3) \\
1(1.3) \\
1(1.3)\end{array}$ \\
\hline Órgano judicial que dicta la resolución & $\begin{array}{l}\text { Audiencia Provincial } \\
\text { Tribunal Superior de Justicia } \\
\text { Juzgado de } 1^{\text {a }} \text { Instancia }\end{array}$ & $\begin{array}{l}72(96) \\
2(2.7) \\
1(1.3)\end{array}$ \\
\hline Órgano judicial de procedencia & $\begin{array}{l}\text { Juzgado de } 1^{\text {a }} \text { Instancia } \\
\text { Juzgado de Violencia sobre la Mujer } \\
\text { Audiencia Provincial }\end{array}$ & $\begin{array}{c}58(77.3) \\
15(20.0) \\
2(2.7)\end{array}$ \\
\hline Asunto principal & $\begin{array}{l}\text { Divorcio } \\
\text { Modificación de medidas } \\
\text { Guarda y custodia } \\
\text { Oposición a la ejecución de sentencia } \\
\text { Visitas con abuelos } \\
\text { Recurso de casación } \\
\text { Filiación } \\
\text { Otros }\end{array}$ & $\begin{array}{l}26(34.7) \\
24(32.0) \\
14(18.7) \\
2(2.7) \\
3(4.0) \\
2(2.7) \\
1(1.2) \\
3(4.0)\end{array}$ \\
\hline
\end{tabular}




\section{Análisis de los protocolos}

Para la creación del sistema categorial metódico de análisis de contenido (Sánchez, Sobral y Seijo, 2017), de las sentencias (protocolos), siguiendo para ello las directrices de Arce, Fariña y Fraga (2000). Se partió del sistema de categorías iniciales de análisis de contenido de sentencias judiciales que motivaban la decisión en casos de familia (Arce, Fariña y Seijo, 2005); con categorías de motivación de la decisión legal y de hecho (Arce, Tortosa, y Alfaro, 2003); y con un procedimiento de búsqueda de nuevas categorías por aproximaciones sucesivas (Fariña, Arce y Novo, 2002). Asimismo, se tomaron las directrices de la AFCC (2006) y la APA (2012) para la creación de categorías de contenido sobre cómo establecen jueces y magistrados la práctica de la CP. Se creó un manual con las definiciones y ejemplos de cada categoría, entrenándose los codificadores con otro material no incluido en el estudio. En concreto, las categorías de análisis productivas, así como una definición de las mismas se relacionan seguidamente:

1. Motivación legal de las sentencias que contemplan la $C P$. Se codifica la fundamentación legal que aparece en los protocolos que incluyen la $\mathrm{CP}$, teniendo en cuenta si se trata de leyes internacionales, nacionales o autonómicas, así como la jurisprudencia.

2. Motivación de la práctica del coordinador parental. Esta dimensión se compone a su vez de distintos criterios:

a. Objeto de la intervención. Describe las distintas funciones atribuidas al CP, concretamente: (a) Normalizar el sistema de custodia y régimen de estancias y comunicaciones; (b) Normalizar o restablecer o ayudar a mantener la relación de coparentalidad; (c) Mejora de habilidades; (d) Otras (por ejemplo, seguimiento y apoyo, proponer o concretar el régimen de estancias y comunicaciones, o cualquier otro objetivo).

b. Formación del CP. Hace referencia a la profesión o formación de origen de los coordinadores de parentalidad que señala la resolución judicial.

c. Funciones del CP. Hace alusión a las funciones del CP que se recogen en la resolución judicial, pudiendo diferenciarse entre: (a) Educación, (b)
Evaluación, (c) Gestión del caso, d) Gestión de conflictos, y (e) Toma de decisiones.

d. Designación del CP. Este criterio da cuenta de si se produce la designación del $\mathrm{CP}$, del modo en cómo se produce la misma, es decir si el CP es ordenado por el juez y si es acordado por los propios progenitores; $\mathrm{y}$, si la designación del $\mathrm{CP}$ se realiza a determinadas entidades o servicios.

e. Período de intervención de la CP. Se examina si la resolución judicial establece la duración que debe tener la intervención del coordinador.

f. Comunicación y seguimiento con el órgano judicial. Este criterio describe si el coordinador designado para intervenir con la familia debe enviar informes de comunicación y seguimiento al Juzgado.

\section{Fiabilidad de la Codificación}

El análisis de contenido de las sentencias de la codificación se llevó a cabo siguiendo el procedimiento de otros estudios (Arce et al., 2002). Se procedió al cálculo de la fiabilidad intra-codificadores, inter-codificadores e inter-contextos (otros estudios; Fariña et al., 2005). Como criterio de consistencia se tomó la concordancia, considerándose consistente la codificación si ésta es superior a .80 (Tversky, 1977). Adicionalmente, se verificó la exacta correspondencia entre las codificaciones, no el recuento (Arce et al., 2002). De este modo la consistencia es más restrictiva que el índice Kappa. Cada codificador analizó, de modo independiente, todos los protocolos del estudio (se tomaron para el análisis de datos aleatoriamente la mitad de cada uno de ellos, pares del codificador 1 e impares del 2) y el $20 \%$ de los protocolos transcurrida una semana de la codificación original. Los resultados mostraron índices de concordancia inter- e intracodificadores superiores a .80 . En suma, los resultados son consistentes inter- e intra-codificador, así como con otros codificadores en otros contextos; esto es, fiables (Wicker, 1975).

\section{Análisis de datos}

Se llevaron a cabo análisis descriptivos de frecuencias para obtener los datos en relación a la motivación legal 
del CP. Para estimar la prevalencia de los criterios establecidos en relación a la figura del $\mathrm{CP}$ y su intervención en cuanto a las dimensiones de formación, funciones, designación, objeto de la intervención, duración e informes de seguimiento con el juzgado, se realizaron análisis descriptivos de frecuencias y porcentajes. Como criterio de evaluación de la prevalencia se tomó el de incidencia significativa; esto es, en línea con el criterio de Arce et al. (2002) para establecer si una continencia es representativa de una determinada condición, se tomó la probabilidad máxima de prevalencia por azar (.05) con valor de contraste, de modo que las contingencias con una tasa de manifestación significativamente superior a 0.5 están más representadas en las sentencias judiciales de lo esperado por azar y, por tanto, son características de la condición de las sentencias sobre coordinación de parentali- dad. Como tamaño del efecto se calculó la $h$ de Cohen, que es equivalente a la $d$ para el contraste de proporciones $(h=0.20$, tamaño pequeño; $h=0.50$ tamaño moderado; $h=0.80$, tamaño grande del efecto).

\section{Resultados}

\section{De la motivación legal en las resoluciones ju- diciales que contemplan la Coordinación de Parentalidad}

Los resultados obtenidos muestran que los jueces y magistrados hacen referencia principalmente en las sentencias que incluyen la $\mathrm{CP}$, al Código Civil (8\%), a la

Tabla 2

Motivación legal en las resoluciones judiciales que contemplan el coordinador de parentalidad

\begin{tabular}{|c|c|c|}
\hline $\begin{array}{r}\text { Fuente de Derecho } \\
\end{array}$ & $f(\%)$ & Detalles legales \\
\hline Constitución Española & $2(2.6)$ & 39 \\
\hline Código Civil & $6(8.0)$ & $92 ; 158$ \\
\hline $\begin{array}{l}\text { Ley Orgánica 1/1996, de } 15 \text { de enero, de } \\
\text { Protección Jurídica del Menor. }\end{array}$ & $3(4.0)$ & Disposición Adicional $1^{a}$ \\
\hline $\begin{array}{l}\text { Ley } 1 / 2000 \text {, de } 7 \text { de enero, de Enjuiciamiento } \\
\text { Civil. }\end{array}$ & $7(9.3)$ & $\begin{array}{l}\text { 241; 335; 341; 748; 749; 750; 751; 752; 753; 754; 755; } \\
770 ; 776\end{array}$ \\
\hline $\begin{array}{l}\text { Ley } 15 / 2005 \text {, de } 8 \text { de julio, en materia de } \\
\text { separación y divorcio. }\end{array}$ & $1(1.3)$ & - \\
\hline Estatuto de Autonomía de Cataluña. & $2(2.6)$ & 17 \\
\hline $\begin{array}{l}\text { Ley } 25 / 2010 \text {, de } 29 \text { de julio, del libro segundo del } \\
\text { Código Civil de Cataluña. }\end{array}$ & $32(42.66)$ & $\begin{array}{l}\text { 211-6; 233-10; 233-12; 233-13; 233-14; 236-3; 236-4; } \\
\text { 236-6; 236-13; } \\
\text { Disposición Adicional 6a } \\
\text { Disposición Adicional 7a }\end{array}$ \\
\hline $\begin{array}{l}\text { La ley } 14 / 2010 \text {, de } 27 \text { de mayo, de los derechos y } \\
\text { las oportunidades en la infancia y la adolescencia }\end{array}$ & $3(4.0)$ & 12 \\
\hline $\begin{array}{l}\text { Convención sobre los Derechos del Niño } \\
\text { adoptada por la Asamblea General de las } \\
\text { Naciones Unidas el } 20 \text { de noviembre de } 1989 \text {. }\end{array}$ & $2(2.6)$ & $3 ; 9$ \\
\hline $\begin{array}{l}\text { Recomendación } \operatorname{Rec}(2006) 19 \text { del Comité de } \\
\text { Ministros a los Estados Miembros sobre políticas } \\
\text { de apoyo al ejercicio positivo de la parentalidad }\end{array}$ & $2(2.6)$ & - \\
\hline \multirow[t]{4}{*}{ Jurisprudencia. } & $\begin{array}{l}3(4.0) \\
6(8.0)\end{array}$ & $\begin{array}{l}\text { STSJC de } 16 \text { de junio de } 2011 . \\
\text { TSJC, } 26 \text { de febrero } 2015 .\end{array}$ \\
\hline & $2(2.6)$ & $\begin{array}{l}\text { STC } 4 / 2001 \text {, de } 15 \text { de enero; } 58 / 2008 \text {, de } 28 \text { de abril, o } \\
\text { 185/2012, de } 17 \text { de octubre. }\end{array}$ \\
\hline & $\begin{array}{l}3(4.0) \\
2(26)\end{array}$ & STS de 7 de abril de 2011 y de 5 de octubre de 2011 . \\
\hline & $1(1.3)$ & $\begin{array}{l}\text { TEDH de } 22 \text { de junio de } 2006 \text { (Bianchi contra Suiza), } 2 \\
\text { de septiembre de } 2010 \text { (Mincheva contra Bulgaria) y } 29 \\
\text { de enero de } 2013 \text { (Lombardo contra Italia). }\end{array}$ \\
\hline
\end{tabular}

Nota. $f(\%)$ : frecuencia(porcentaje). 
Ley 1/2000 de Enjuiciamiento Civil (9.3\%), y de forma mayoritaria a la Ley $25 / 2010$, de 29 de julio, del libro segundo del Código Civil de Cataluña (42.66\%). Por otra parte, atendiendo a la jurisprudencia, las resoluciones judiciales analizadas (véase Tabla 2) se apoyan en mayor medida en la sentencia del TSJC de 26 febrero de 2015 (8\%); las sentencias del TS de 7 de abril de 2011 y de 5 de octubre de 2011 (6\%); y la sentencia del TSJC de 16 de junio de 2011 (4\%).

\section{De la motivación para la orientación de la práctica del coordinador parental}

Del total de seis criterios de la práctica del CP (relativos a la formación, a las funciones, a la designación, al objeto de la intervención, a la duración y a los informes de seguimiento al juzgado), los resultados indican (véase Tabla 3) que un $30.66 \%$ de las sentencias analizadas recogen tres criterios y un $25.34 \%$, un total de cuatro. Ninguna de las sentencias recoge los seis criterios y tres de ellas $(4 \%)$ no introduce ninguno. El número de criterios promedio en las sentencias analizadas es de 2.7, con un rango de cero a seis. Por otra parte, de los criterios establecidos en las directrices internacionales, los resultados muestran que los relativos al objeto de la intervención del CP $(90.66 \%)$, a sus funciones $(60.0 \%)$ y a su designación $(57.33 \%)$, son los que están más presentes en las sentencias analizadas. Con menor impacto, el criterio de seguimiento por el juzgado $(30.60 \%)$, la forma- ción del coordinador parental $(22.66 \%)$ y la duración de la intervención (10.66\%).

A su vez, en la Tabla 4, se presenta la productividad de cada criterio atendiendo a la significación estadística. Así, en cuanto al objeto de la intervención del CP, las categorías que resultan significativamente productivas son la normalización o el restablecimiento de la relación de los hijos con los progenitores y la mejora o ayuda a mantener la relación de coparentalidad entre ambos progenitores. Por otro lado, en relación a las funciones del CP referidas en las resoluciones judiciales resultan productivas todas las funciones: gestión del conflicto, evaluación, gestión del caso y educativa, con excepción de la toma decisiones. Por su parte, en cuanto a los criterios de formación del CP, o bien no se especifica o se establece la formación en mediación familiar.

Por último, tal y como se refleja en la Tabla 4, son criterios también productivos la designación del $C P$ en resolución judicial, a su vez, realizada por el tribunal, centro de mediación, progenitores, o del listado del colegio oficial de psicólogos. De igual modo, se incluye entre los criterios con impacto en las sentencias, la no especificación de la duración de la intervención del CP, ni del modo de seguimiento del caso con el juzgado, si bien cuando este se concreta, se establece para tres meses o menos, o de cuatro a seis.

\section{Tabla 3}

Frecuencia y porcentaje de criterios del coordinador de parentalidad en las resoluciones judiciales

\begin{tabular}{|c|c|}
\hline Número de criterios & $f(\%)$ \\
\hline 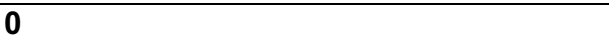 & $3(4.0)$ \\
\hline 1 & $10(13.34)$ \\
\hline 2 & $17(22.66)$ \\
\hline 3 & $23(30.66)$ \\
\hline 4 & $19(25.34)$ \\
\hline 5 & $3(4.0)$ \\
\hline Total & $75(100)$ \\
\hline
\end{tabular}

Nota. $f(\%)$ : frecuencia(porcentaje). 
Tabla 4

Criterios para la motivación de la orientación de la práctica del coordinador de parentalidad

\begin{tabular}{|c|c|c|c|c|c|}
\hline & & & $f(p)$ & $Z(.05)+$ & $\boldsymbol{h}$ \\
\hline \multirow{4}{*}{$\begin{array}{l}\text { Objeto } \\
(n=75)\end{array}$} & \multicolumn{2}{|c|}{ Normalizar o restablecer la relación de los hijos con los progenitores } & $24(.32)$ & $10.8^{* * *}$ & 1.18 \\
\hline & \multicolumn{2}{|c|}{ Mejorar o ayudar a mantener la relación de coparentalidad } & $30(.40)$ & $14^{* * *}$ & 1.39 \\
\hline & \multicolumn{2}{|c|}{ Mejora de habilidades de los progenitores } & $7(.09)$ & 1.72 & 0,30 \\
\hline & \multicolumn{2}{|l|}{ Otros objetivos } & $6(.08)$ & 0.39 & 0.24 \\
\hline Formación & Ninguna & & $57(.76)$ & $28.4^{* * *}$ & 2.35 \\
\hline \multirow[t]{3}{*}{$(n=75)$} & \multicolumn{2}{|l|}{ Mediador familiar } & $9(.12)$ & $2.8^{* *}$ & 0.47 \\
\hline & \multicolumn{2}{|l|}{ Psicólogo } & $5(.06)$ & 0.68 & 0.09 \\
\hline & \multicolumn{2}{|c|}{ Perito especializado en conflicto o coordinación de parentalidad } & $4(.05)$ & 0.12 & 0.00 \\
\hline Funciones & \multicolumn{2}{|l|}{ Gestión del conflicto } & $39(.52)$ & $18.8^{* * *}$ & 1.70 \\
\hline \multirow[t]{4}{*}{$(n=75)$} & \multicolumn{2}{|l|}{ Evaluación } & $26(.34)$ & $11.84^{* * *}$ & 1.23 \\
\hline & \multicolumn{2}{|l|}{ Gestión del caso } & $19(.25)$ & $8.12^{* * *}$ & 0.97 \\
\hline & \multicolumn{2}{|l|}{ Educativa } & $15(.20)$ & $6.00^{* * *}$ & 0.80 \\
\hline & \multicolumn{2}{|l|}{ Toma de decisiones } & $6(.08)$ & 1.2 & 0.24 \\
\hline Designación & \multicolumn{2}{|c|}{ Se designa al CP en la resolución judicial } & $60(.80)$ & $26.78^{* * *}$ & 2.49 \\
\hline \multirow[t]{9}{*}{$(n=60)$} & Quien & Tribunal & $38(.63)$ & $26.82^{* * *}$ & 1.98 \\
\hline & $(n=60)$ & Progenitores & $16(.26)$ & $7.75^{\star * *}$ & 1.00 \\
\hline & \multirow{7}{*}{$\begin{array}{l}\text { A quien se designa } \\
(n=60)\end{array}$} & Fiscal & $3(.05)$ & 0.00 & 0.00 \\
\hline & & \multirow{2}{*}{$\begin{array}{l}\text { Otra institución } \\
\text { Centro de Mediación de la Generalitat de Cataluña }\end{array}$} & $3(.05)$ & 0.00 & 0.00 \\
\hline & & & $30(.50)$ & $16.07^{* * *}$ & 1.64 \\
\hline & & Lista de Colegio de Psicólogos de Cataluña & $13(.21)$ & $5.92^{* * *}$ & 0.84 \\
\hline & & Inespecífico & $6(.10)$ & 1.78 & 0.36 \\
\hline & & Servicios Sociales & $6(.10)$ & 1.78 & 0.36 \\
\hline & & Otros & $5(.08)$ & 1.21 & 0.24 \\
\hline Duración & \multicolumn{2}{|l|}{ No se especifica } & $64(.85)$ & $32.16^{\star * *}$ & 2.68 \\
\hline \multirow[t]{3}{*}{$(n=75)$} & \multicolumn{2}{|l|}{ Dos o tres meses } & $6(.08)$ & 1.2 & 0.24 \\
\hline & \multicolumn{2}{|c|}{ De cuatro a seis meses } & $2(.02)$ & -0.96 & -0.41 \\
\hline & \multicolumn{2}{|c|}{ El tiempo necesario para asegurar la estabilidad de los menores } & $3(.04)$ & -0.4 & -0.11 \\
\hline Seguimiento & \multicolumn{2}{|l|}{ No se establece } & $52(.69)$ & $25.72^{\star * *}$ & 2.15 \\
\hline$(n=75)$ & \multicolumn{2}{|l|}{ Tres meses o menos } & $15(.20)$ & $6^{* * *}$ & 0.80 \\
\hline & \multicolumn{2}{|c|}{ De cuatro a seis meses } & $8(.10)$ & $2.28^{*}$ & 0.36 \\
\hline
\end{tabular}




\section{Discusión}

Con carácter previo, hemos de señalar las limitaciones de este trabajo, en primer lugar, este al centrarse exclusivamente en las resoluciones judiciales, adolece de falta de generalización a toda la tarea judicial (Arce, Fariña y Seijo, 2005). En segundo lugar, a pesar de que el estudio contó con el total de las sentencias dictadas en España sobre $\mathrm{CP}$, el tamaño de la muestra es reducido, debido a que la $\mathrm{CP}$ es una figura profesional todavía con escasa trayectoria y poco conocida en España, estando principalmente instaurada en Cataluña. Teniendo presentes estas consideraciones, se puede concluir, en base a los resultados alcanzados, que las sentencias en las que se incluye la figura del CP cuentan con motivación legal, dando cumplimiento al art. 24 de la Constitución Española que establece que toda resolución judicial ha de estar motivada. Para ello, los decisores judiciales se amparan en normativa legal y jurisprudencia de carácter nacional, autonómico y europeo. Además, este estudio permite describir la práctica del coordinador parental en las resoluciones judiciales. Así, los jueces y magistrados españoles aplican mayoritariamente algún criterio que se corresponde con las directrices establecidas internacionalmente por la AFCC (2005) y la APA (2012), con referencia a la formación, a las funciones, a la designación, al objeto de la intervención, a la duración y a los informes de seguimiento al juzgado, siendo los más utilizados el objeto de la intervención, sus funciones y la designación del CP. Para establecer un mandato de intervención de $\mathrm{CP}$ estos resultan esenciales, pero parecen insuficientes para enmarcar su aplicación en cada caso concreto, máxime tratándose de una nueva figura profesional en nuestro país (Capdevila, 2016). En este sentido, es de resaltar que el $69 \%$ de las resoluciones judiciales no hacen referencia a cómo se ha de establecer el seguimiento judicial de la intervención, ni tampoco se limita el período que durará la misma en el $85 \%$ de ellas, a pesar de que la duración no debería ser inferior a un año (Fariña et al., 2017), así muchos tribunales la limitan a uno o dos años (Carter, 2011). También es importante indicar que los decisores judiciales dirigen la labor del $\mathrm{CP}$ a la gestión del conflicto, a la evaluación, a la gestión del caso y en menor medida a la función educativa, mientras que la toma de decisiones no es un criterio productivo, apareciendo únicamente en el $8 \%$ de las sentencias analizadas, cuando esta es una de las funciones primordiales del CP para cuestiones del día a día en relación a la coparentalidad (Carter, 2011; Emery et al., 2014). El CP tiene que tener autoridad para arbitrar y tomar decisiones cuando los progenitores no son capaces de llegar a acuerdos (Demby, 2016) y la situación requiere una respuesta. Es más, parece demostrado que la autoridad limitada del CP reduce la eficacia y la satisfacción de la mayoría de los usuarios (Demby, 2016; Mandarino et al., 2016). De esta manera, cuando no existen normas legales que regulen la figura del CP, tal es el caso de España, y se quiera designar judicialmente a un CP con autoridad para tomar decisiones, se ha de especificar en el nombramiento que se otorga suficiente autoridad (Montiel, 2015), y como, el mismo autor concreta, sin que ello signifique delegar la función judicial.

De lo expuesto anteriormente, podemos señalar que, aunque los criterios que manejan los decisores judiciales se corresponden, en lo esencial, con las directrices editadas por las dos asociaciones internacionales, sería preciso que los operadores jurídicos, principalmente jueces, fiscales, letrados de la administración de justicia y abogados pudieran conocer mejor las posibilidades que tiene la $\mathrm{CP}$, para ayudar a las familias con separaciones conflictivas de los progenitores para que puedan ejercer una coparentalidad positiva, velando por el interés de sus hijos; así como comprender bien el rol del CP. Es sabido que los beneficios de poner en práctica un servicio de $\mathrm{CP}$ para aquellos progenitores que no son capaces de comunicarse de manera efectiva si no es a través de una relación de litigio continuado, han sido comprobados (Brewster, Beck, Anderson y Benjamin, 2011; Fidler, 2012; Fieldstone et al., 2011; Henry et al., 2009; Kelly, 2002; Neff y Cooper, 2004; Parks, Tindall y Yingling, 2011). Por eso en Estados Unidos y Canadá, los jueces recurren cada vez más a la CP para proporcionar a estos progenitores la orientación, la educación y las habilidades de resolución de problemas necesarias para asumir sus responsabilidades parentales (D'Abate, 2016). Y los CP intentan ofrecer las respuestas más ajustadas a las necesidades de las familias, para ello se han desarrollado modalidades diferentes del planteamiento original de la práctica de la $\mathrm{CP}$, como por ejemplo el Modelo de dos Coordinadores Parentales (Behrman, 2016). De este modo, la CP se convierte en un recurso altamente especiali- 
zado, enmarcado dentro de los que ofrece el paradigma de la Justicia Terapéutica (Fariña et al., 2017), para proteger el bienestar de la infancia y la adolescencia, a la vez que la de sus progenitores y familiares próximos, y a la sociedad en general. No se puede obviar que cuanto más tiempo permanece un caso en el sistema judicial, mayor es el coste no sólo para este, sino también para la familia y la comunidad (D’Abate, 2016; Henry et al., 2009).

\section{Referencias}

American Bar Association. (2005). High conflict Custody Cases: Reforming the System for Children. Family Court Review, 39, 146-157. https://doi.org/10.1111/j.174-1617.2001.tb00600.x

American Psychological Association. (2012). Guidelines for the Practice of Parenting Coordination. The American Psychologist, 67, 6371. https://doi.org/10.1037/a0024646

Arce, R., Fariña, F. y Fraga, A. (2000). Género y formación de juicios en un caso de violación [Gender and Juror Judgment Making in a Case of Rape]. Psicothema, 12(4), 623-628. Recuperado de http://www.psicothema.com/pdf/381.pdf

Arce, R., Fariña, F. y Seijo, D. (2005). Razonamientos judiciales en procesos de separación [Judicial Reasoning in Parental Separation and Divorce Proceedings: Content and Cognitive Analysis of Judicial Reasoning]. Psicothema, 17, 57-63. Recuperado de http://www.psicothema.com/pdf/3064.pdf

Arce, R., Tortosa, F. y Alfaro, E. (2003). Veredictos y análisis del contenido de las deliberaciones de los Tribunales de Jueces y Jurados en el contexto jurídico español [Verdict and Deliberation Content Analysis of Judges and Juries in the Spanish Legal Context. A Contrastive Approach]. Psicothema, 15(1), 127-135. Recuperado de http://www.psicothema.es/pdf/1034.pdf
Association of Family \& Conciliation Courts. (2006). Guidelines for Parenting Coordination. Family Court Review, 44, 164-181. https://doi.org/10.1111/j.1744-1617.2006.00074.x

Avedillo, M., Carrasco, L., Guitart, E. y Sacasas, M. (2015). La coordinación de parentalidad: Cuando las familias ya no saben qué hacer [Parenthood Coordination. When Families no Longer Know what to Do]. Barcelona, España: Huygens.

Behrman, L. (2016). It takes a Village-Taming high Conflict with the "2 PC Model". Journal of Clinical Psychology: In Session, 72, 469-483. https://doi.org/10.1002/jclp.22257

Boyan, S. y Termini, A. M. (2005). The Psychotherapist as a Parenting Coordinator in High Conflict Divorce. New York, NY: Hawthorn Press.

Brewster, K., Beck, C., Anderson, E. y Benjamin, A. (2011). Encouraging Results from Pilot Testing a Research Methodology. Journal of Child Custody, 8 , 247-267. https:/doi.org/10.1080/15379418.2011.620926

Brown, W. R., Behrman, L. y Zimmerman, J. (2017). Duel or dual: An Interdisciplinary approach to Parenting Coordination for Uber-Conflicted Parenting Relationships. Family Court Review, 55, 345-361. https://doi.org/10.1111/fcre.12284

Capdevila, C. (2016). La coordinación de coparentalidad. Una intervención especializada para familias en situación de alta conflictividad crónica post-ruptura de pareja [The Coparenting Coordination. A Highly Specialized Intervention for Families in chronic High Conflict PostSeparation]. Anuario de Psicología, 46, 41-49. https://doi.org/10.1016/j.anpsic.2016.06.005

Carter, D. K. (2011). Parenting Coordination: The integrated model. En D. K. Carter (Ed.), Parenting coordination: A Practical Guide for Family Law 
Professionals (pp. 1-17). New York, NY: Springer Publishing Company.

Coates, C. A., Deutsch, R., Starnes, H., Sullivan, M. J. y Sydlik, B. (2004). Parenting Coordination for High-Conflict Families. Family Court Review, 42, 246-262. https://doi.org/10.1111/j.1741617.2004.tb00647.x

Consejo de Europa. (2015). Resolución de la Asamblea Parlamentaria del Consejo de Europa 2079/2015 sobre igualdad y corresponsabilidad. Recuperado de http://assembly.coe.int/nw/xml/XRef/XrefDocDetails-EN.asp?FileID $=22022$ \&lang $=E N$

Corrás, T., Seijo, D., Fariña, F., Novo, M., Arce, R. y Cabanach, R. G. (2017). What and how much Do Children Lose in Academic Settings Owing to Parental Separation? Frontiers in Psychology, 8, 1545. https://doi.org/10.3389/fpsyg.2017.01545

D'Abate, D. (2016). Use of Solution-Focused and Family Narrative Approaches in Working with High Conflict Families: Strategies and Techniques that Can Be Utilized in Parenting Coordination and Co-Parenting Coaching. Journal of Child Custody, 13, 269-288. https://doi.org/10.1080/15379418.2016.1247308

D'Abate, D. (2005). Parenting Coordination: A New Service for High Conflict Divorcing Families. Intervention OPTSQ, 122, 1-9. Recuperado de http://www.adrmaremma.it/english/dabate01.pdf

Demby, S. (2016). Parenting Coordination: Applying Clinical Thinking to the Management and Resolution of Post-Divorce Conflict. Journal of Clinical Psychology, 72, 458-468. https://doi.org/10.1002/jclp.22261

Deutsch, R. M. (2014). Parenting Coordination: Basic Approaches and Strategies. En S. A. Higuchy y S. J. Lally (Eds.), Parenting Coordination in Post Separation Disputes: A Comprehensive Guide for Practitioners (pp. 63-74). Washington, DC: American Psychological Association.
Deutsch, R. M. y Lally, S. J. (2014). Ethical Issues and Risk Management in Parenting Coordination. En S. A. Higuchy y S. J. Lally (Eds.), Parenting Coordination in Post Separation Disputes: A Comprehensive Guide for Practitioners (pp. 93106). Washington, DC: American Psychological Association.

Emery, R. E., Rowen, J. y Dinescu, D. (2014). New Roles for Family Therapists in the Courts: An Overview with a Focus on Custody Dispute Resolution. Family Process, 53, 500-515. https://doi.org/10.1111/famp.12077

Fariña, F., Arce, R. y Novo, M. (2002). Heurístico de anclaje en las decisiones judiciales [Anchorage in Judicial Decision Making]. Psicothema, 14(1), 3946. Recuperado de http://www.psicothema.com/pdf/684.pdf

Fariña, F., Novo, M., Arce, R. y Vázquez, M. J. (2017). Intervenciones con familias tras la ruptura de pareja con enfoque de Justicia Terapéutica: Programas de apoyo y coordinación de parentalidad [Interventions with Families after the Break-Up with a Therapeutic Justice Approach: Support Programmes and Parental Care Coordination]. En H. Marchiori (Dir.), Victimología (pp. 25-46). Córdoba, Argentina: Encuentro Grupo Editor.

Fidler, B. J. (2012). Parenting Coordination: Lessons Learned and Key Practice Issues. Canadian Family Law Quarterly, 3, 237-273.

Fieldstone, L., Carter, D. K., King, T. y McHale, J. P. (2011). Training, Skills, and Practices of Parenting Coordinators: Florida Statewide Study. Family Court Review, 49, 801-817. https://doi.org/10.1111/j.1744-1617.2011.01415.x

Henry, W. J., Fieldstone, L. y Bohac, K. (2009). The Impact of Parenting Coordination on Court Relitigation: A Case Study. Family Court Review, 47, 682-697. https://doi.org/10.1111/j.17441617.2009.01281.x 
Henry, W. J., Mitcham, M. A. y Henry, L. M. (2013). Conflict Resolution Strategies Adopted from Parenting Coordination: Assisting High-conflict Coparenting Students. Journal of College Counseling, 16, 176-190. https://doi.org/10.1002/j.2161-1882.2013.00035.x

Kelly, J. B. (2002). Psychological and Legal Interventions for Parents and Children in Custody and Access Disputes: Current Research and Practice. Virginia Journal of Social Policy \& the Law, 10, 129-163.

Kelly, J. B. (2003). Parents with Enduring Child Disputes: Multiple Pathways to Enduring Disputes. Journal of Family Studies, 9, 37-50. https://doi.org/10.5172/jfs.9.1.37

Kelly, J. B. (2014). Including Children in the Parenting Coordination Process: A Specialized Role. En S. A. Higuchy y S. J. Lally (Eds.), Parenting Coordination in Post Separation Disputes: A Comprehensive Guide for Practitioners (pp. 143170). Washington, DC: American Psychological Association.

Kirkland, K. y Sullivan, M. (2008). Parenting Coordination (PC) Practice: A Survey of Experienced Professionals. Family Court Review, $46, \quad 622-636$. https://doi.org/10.1111/j.17441617.2008.00228.x

Lally, S. y Deutsch, R. (2014). Competencies Required for Conducting Parenting Coordination. En S. A. Higuchi y S. J. Lally (Eds.), Parenting Coordination in Postseparation Disputes: A Comprehensive Guide for Practitioners (pp. 4361). Washington, DC: American Psychological Association.

Mandarino, K., Kline, M. y Fieldstone, L. (2016). Coparenting in a Highly Conflicted separation/divorce: Learning about Parents and their Experiences of Parenting Coordination, Legal, and Mental Health Interventions. Family
Court Review, 54, 564-577. https://doi.org/10.1111/fcre.12243

Martinón, J. M., Fariña, F., Corrás, T., Seijo, D., Souto, A. y Novo, M. (2017). Impacto de la ruptura de los progenitores en el estado de salud física de los hijos [Impact of Parental Breakup on the Physical Health of Children]. European Journal of Education and Psychology, 10, 9-14. https://doi.org/10.1016/j.ejeps.2016.10.002

Mitcham-Smith, M. y Henry, W. J. (2007). HighConflict Divorce Solutions: Parenting Coordination as an Innovative Co-Parenting Intervention. The Family Journal: Counseling and Therapy for Couples and Families, 15, 368-373. https://doi.org/10.1177/1066480707303751

Montiel, J. T. (2015). Out on a limb: Appointing a parenting coordinator with decision making authority in the absence of a statute or rule. Family Court Review, 53, 632-649. https://doi.org/10.1111/fcre.12174

Neff, R. y Cooper, K. (2004). Parental Conflict Resolution: Six, Twelve, and Fifteen-Month Follow-Ups of a High-Conflict Program. Family Court Review 42, 99-114. https://doi.org/10.1111/j.174-1617.2004.tb00636.x

Ordway, A. M. (2015). Parenting Coordination: A New Frontier for Professional Counselors. VISTAS Online, $30 . \quad$ Recuperado de https://www.counseling.org/docs/defaultsource/vistas/parenting-coordination-a-newfrontier-for-professionalcounselors.pdf?sfvrsn $=$ da4a412c_10

Ortuño, P. (2013). La supervisión de las relaciones parentales tras la sentencia judicial. Familia $y$ Sucesiones [Supervision of Parental Relations after the Court Judgement. Family and Succession]. Cuaderno Jurídico, 107, 20-26.

Parks, L. S., Tindall, H. L. y Yingling, L. C. (2011). Defining Parenting Coordination with State Laws. 
Family Court Review, 49, 629-641. https://doi.org/10.1111/j.1744-1617.2011.01398.x

Rodríguez-Domínguez, C. y Carbonell, X. (2014). Coordinador de parentalidad: Nueva figura profesional para el psicólogo forense [Parentality Coordinator: New Professional Figure for the Forensic Psychologist]. Papeles del Psicólogo, 35, 193-200.

Saini, M. y Birnbaum, R. (2007). Unraveling the Label of High Conflict: What Factors Really Count in Separated and Divorced Families? Ontario Association of Children's Aid Societies Journal, 51, 14-20.

Sánchez, N., Sobral, J. y Seijo, D. (2017). El error judicial en el uso de la prisión preventiva: Personas en prisión que nunca llegan a ser condenadas [Judicial Error in Preventive Prison: People in Prison who Will never Be Convicted]. Revista Iberoamericana de Psicología y Salud, 8, 36-43. https://doi.org/10.23923/j.rips.2017.08.004

Shaw, M. (2017). Commentary Regarding Parenting Coordination in Cases of High Conflict Disputes. Journal of Child Custody, 14, 7376. https://doi.org/10.1080/15379418.2017.1286961

Seijo, D., Fariña, F., Corras, T., Novo, M. y Arce, R. (2016). Estimating the Epidemiology and Quantifying the Damages of Parental Separation in Children and Adolescents. Frontiers in Psychology, $7, \quad 1611$. https://doi.org/10.3389/fpsyg.2016.01611

Sullivan, M. J. (2013). Parenting Coordination: Coming of Age? Family Court Review, 51, 56-62. https://doi.org/10.1111/fcre.12008

Sullivan, M. J. (2004). Ethical, Legal, and Professional Practice Issues Involved in Acting as a Psychologist Parent Coordinator in Child Custody Cases. Family Court Review, 42, 576-582. http://doi.org/10.1111/j.174-1617.2004.tb00670.x
Tversky, A. (1977). Features of Similarity. Psychological Review, 84, 327-352. https://doi.org/10.1037/0033-295X.84.4.327

Wicker, A. W. (1975). An Application of th MultitraitMultimethod Logic to the Reliability of Observational Records. Personality and Social Psychology Bulletin, 4, 575-579. http://doi.org/10.1177/014616727500100405 